\title{
A System of Shadow Detection and Shadow Removal for High Resolution Remote Sensing Images
}

\author{
G.Gayathri \\ PG student, Department of Computer Science and Engineering, Parisutham Institute of Technology and Science, \\ Thanjavur, Tamilnadu, India.
}

\begin{abstract}
Shadow will occur by sunlight or any light sources. We cannot get clear and quality picture for obtain the shadow in the images. The objective of this paper is detect and removal of shadows play an important role in application of urban high resolution remote sensing images. Object oriented shadow detection and removal methods are used in this paper. Shadow detection is used during the image segmentation. The suspected shadow are extracted by the statistical features. For shadow removal, support vector machine and adaboost classifier based on IOOPL matching could effectively remove the shadow. According to the homogeneous section shadow removal will be performed. Our method can accurately detect shadows from urban high-resolution remote sensing images and can effectively restore shadows with a rate of over $95 \%$.
\end{abstract}

Keywords: Object oriented, IOOPL, shadow detection, homogeneous section, shadow removal

\section{INTRODUCTION}

In urban areas, surface landscapes are quite difficult, with texture. An improved algorithm exists that combines the a great variety of objects and shadows formed by elevated two methods.

objects such as high buildings, bridges, and trees.

Although shadows themselves can be regarded as a type of First, the Shadow areas are estimated according to the useful information in 3-D restoration, building location space coordinates of buildings calculated from digital recognition, and height estimation they can also interfere surface models and the altitude and azimuth of the sun. with the processing and application of high-resolution Then, to accurately identify a shadow, the threshold value remote sensing images. IKONOS, Quick Bird, Geo Eye, is obtained from the estimated grayscale value of the and Resource 3 for the observation of Earth and the rapid shadow areas [3]. However information such as scene and development of some aerial platforms such as air ships and camera altitude is not usually readily available.

unmanned aerial vehicles, there has been an increasing need to analyze high-resolution images for different applications. For example, shadows may cause improper results during change detection.

Accordingly, the detection and removal of shadows play an important role in applications of urban high resolution remote sensing images.

\section{EXISTING SYSTEM}

Existing shadow detection method can be roughly categorised into two groups.

- $\quad$ Model based method.

- $\quad$ Shadow-feature based method.

The first group uses prior information such as scene, moving objectives and camera altitude to construct shadow models. This group of methods is often used in some specific scene conditions such as aerial image analysis and video monitoring.

The second group of methods identifies shadow areas with information such as grey scale, brightness, saturation, and

Consequently, most shadow detection algorithms are based on shadow features. For example, the shadow region appears as a low grayscale value in the image, and the threshold is chosen between two peaks in the grayscale histogram of the image data to separate the shadow from the non-shadow region.

An illuminate invariance model has been used to detect shadows this method can obtain a comparatively complete shadow outline from a complex scene and derive the shadow-free image by using certain neutral interface reflecting assumptions [2]. In a related study, images are converted into different invariant colour spaces (HSV, $\mathrm{HCV}$, YIQ, and $\mathrm{YCbCr}$ ) to obtain shadows with Otsu's algorithm [4]. This can effectively get rid of the false shadows created by vegetation in certain invariant spaces. Based on that work, a successive thresholding scheme was proposed to detect shadows.

A variety of image enhancement methods have been proposed for shadow removal, such as histogram matching, several enhancement methods were analyze to recover shadows, namely, gamma correction, LCC, and histogram matching. 


\section{DEMERITS}

- Images are converted into different invariant colour spaces to obtain shadows.

- $\quad$ This can effectively get rid of the false shadows created by vegetation in certain invariant spaces.

- $\quad$ Less accuracy and efficiency.

\section{PROPOSED SYSTEM}

Due to the shortcomings of pixel-level shadow detection, we propose a new technique: an object-oriented shadow detection and removal method.

First, the shadow features are evaluated through image segmentation, and suspected shadows are detected with the threshold method.

Second, object properties such as spectral features and geometric features are combined with a spatial relationship in which the false shadows. Shadow removal employs a series of steps. We extract the inner and outer outline lines of the boundary of shadows.

The grayscale values of the corresponding points on the inner and outer outline lines are indicated by the innerouter outline profile lines (IOOPLs).

Homogeneous sections are obtained through IOOPL sectional matching. Finally, using the homogeneous sections the relative radiation calibration parameters between the shadow and non-shadow regions are obtained and shadow removal is performed.

\section{SHADOW DETECTION}

Shadows are created because the light source has been blocked by something.

\section{There are two types of shadows}

\section{- $\quad$ Self-shadow}

\section{- Cast shadow}

A self-shadow is the shadow on a subject on the side that is not directly facing the light source.

A cast shadow is the shadow of a subject falling on the surface of another subject because the former subject has blocked the light source. A cast shadow consists of two parts: the umbra and the penumbra.

The umbra is created because the direct light has been completely blocked, while the penumbra is created by something partly blocking the direct light. In this paper, we mainly focus on the shadows in the cast shadow area of the remote sensing images.

\section{A. Image Segmentation Considering Shadow Features}

Images with higher resolution contain richer spatial information. The spectral differences of neighbouring pixels within an object increase gradually.

Pixel-based methods may pay too much attention to the details of an object when processing high resolution images, Making it difficult to obtain overall structural information about the object.

In order to use spatial information to detect shadows, image segmentation is needed. We adopt convexity model (CM) constraints for segmentation [5].

Traditional image segmentation methods are likely to result in insufficient segmentation, which makes it difficult to separate shadows from dark objects. The CM constraints can improve the situation to a certain degree.

\section{B. Detection of Suspected Shadow}

For shadow detection, a properly set threshold can separate shadow from non-shadow without too many pixels being misclassified.

Researchers have used several different methods to find the threshold to accurately separate shadow and nonshadow area.

We attain the threshold according to the histogram of the original image and then find the suspected shadow objects by comparing the threshold and grayscale average of each object obtained in segmentation.

\section{Elimination of False Shadow}

Dark objects may be included in the suspected shadows, so more accurate shadow detection results are needed to eliminate these dark objects.

Rayleigh scattering results in a smaller grayscale difference between a shadow area and non-shadow area in the blue (B) waveband than in the red (R) and green $(G)$ wavebands.

Consequently, for the majority of shadows, the grayscale average at the blue waveband $G b$ is slightly larger than the grayscale average at the green waveband $G_{g}$. Also, the properties of green vegetation itself make $G_{g}$ significantly larger than $G b$, so false shadows from vegetation can be ruled out by comparing the $G b$ and $G_{g}$ of all suspected shadows. Namely, for the object $i$, when $G b+G_{a}<G_{g}, i$ can be defined to be vegetation and be ruled out. $G a$ is the correction parameter determined by the image type. 
International Journal of Advanced Research in Computer and Communication Engineering Vol. 4, Issue 2, February 2015

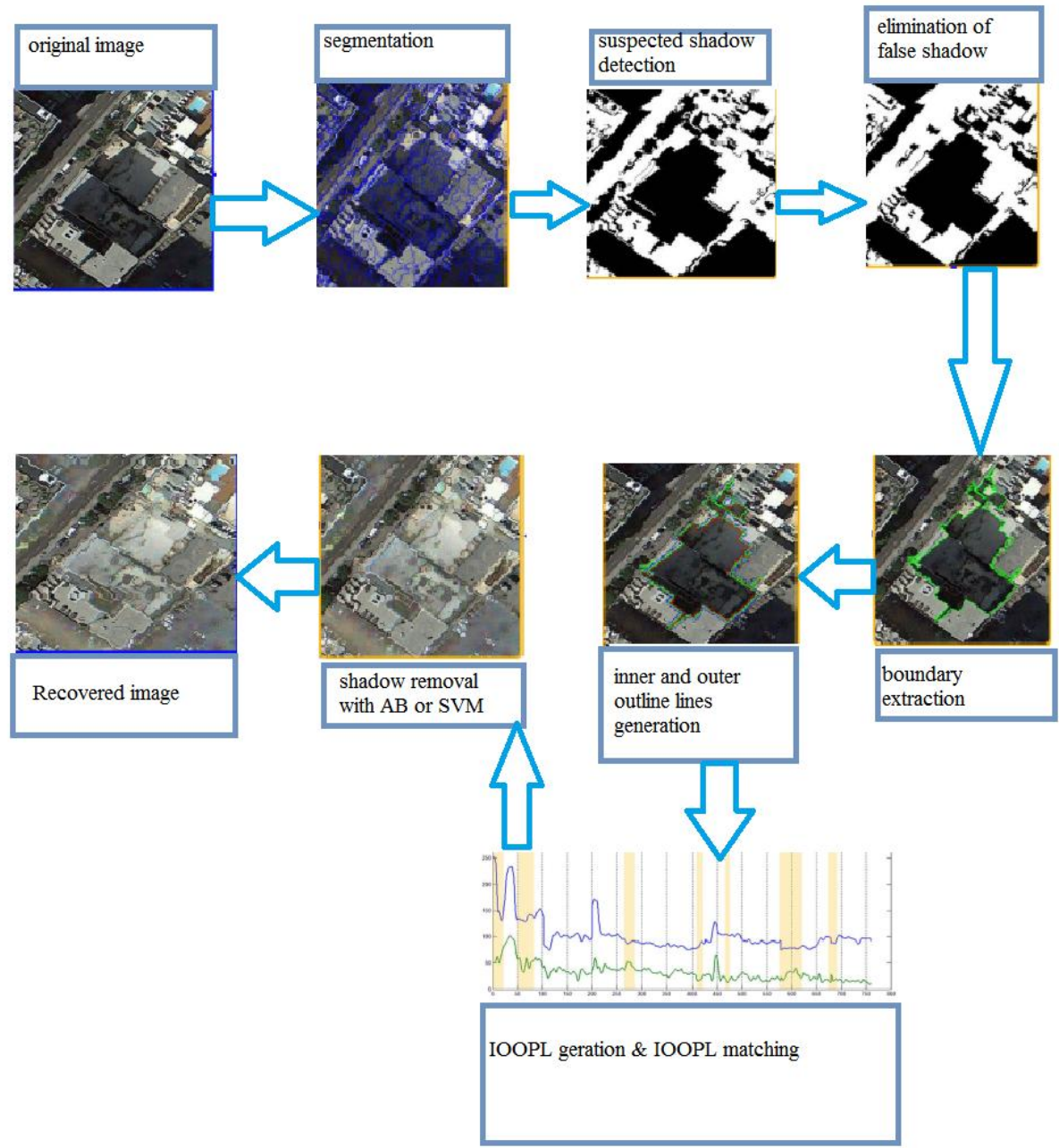

Fig 1: Structure of object-oriented shadow detection and removal from urban high-resolution remote sensing images

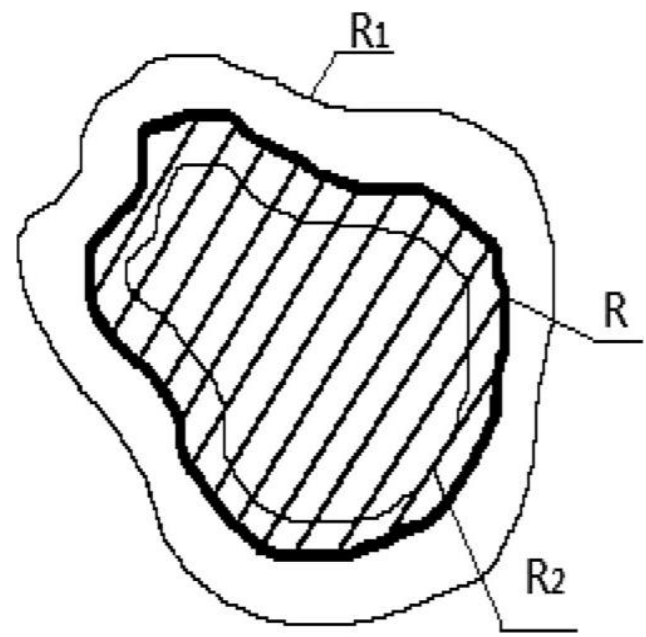

Fig2: Diagram of shadow boundary, inner, and outer outline lines 


\section{SHADOW REMOVAL}

To recover the shadow areas in an image, we use a shadow removal method based on IOOPL matching. There is a large probability that both shadow and non-shadow areas in close range on both sides of the shadow boundary belong to the same type of object. The inner and outer outlines can be obtained by contacting the shadow boundary inward and expanding it outward, respectively. Then, the inner and outer outline profile lines are generated along the inner and outer outline lines to determine the radiation features of the same type of object on both sides.

The objects on both sides of the shadow boundary linked with a building forming a shadow are usually not homogeneous, and the corresponding inner and outer outline profile line sections are not reliable. In addition, the abnormal sections on the inner and outer outlines that cannot represent homogeneous objects need to be ruled out. Consequently, similarity matching needs to be applied to the IOOPL section by section to rule out the two kinds of nonhomogeneous sections mentioned previously. The parameters for shadow removal are obtained by analysing the grayscale distribution characteristics of the inner and outer homogeneous IOOPL sections.

\section{A. IOOPL Matching}

IOOPL matching is a process of obtaining homogeneous sections by conducting similarity the process, Gaussian smoothing is performed to simplify the view of IOOPL.The Gaussian smoothing template parameters [1]. To rule out the non-homogeneous sections, the IOOPL is divided into average sections with the same standard.

\section{B. Implementation of Shadow Removal}

Shadows are removed by using the homogeneous section obtained by line pair matching. There are two approaches for shadow removal. One approach calculates the radiation parameter according to the homogeneous points of each object and then applies the support vector machine to each object. The other approach collects and analyses all the homogeneous sections for Adaboost $(\mathrm{AB})$ and retrieves all shadows directly with the obtained fitting parameters.

\section{1) Adaboost Algorithm}

Boosting is an approach to machine learning based on the idea of creating a highly accurate prediction rule by combining many relatively weak and inaccurate rules.adabost has been widely and rapidly improved for use in pattern recognition. Adaboost linearly combines several classifier in to strong classifier are tuned by minimizing an empirical exponential risk. The classification method exhibits high performance in various field.

\section{- An iterative procedure of adaboost for} minimizing the empirical exponential risk.

Let $\{F\}=\{f: R q \rightarrow G\}$ be a set of classification functions, where $\mathrm{G}=\{1 \ldots \mathrm{g}\}$ is the label set.

Find classification $\mathrm{f}$ in $\mathrm{F}$ and co-efficient $\beta$ that jointly minimize empirical risk $\operatorname{Rexp}(\beta \mathrm{f})$.

Consider empirical risk Rexp $(\beta 1 \mathrm{fl}+\beta \mathrm{f})$ with $\beta 1 \mathrm{fl}$ from the previous step then find classification function $f \varepsilon \mid F 1$ and coefficient $\beta$ that minimize the empirical risk.

This procedure is repeated T-times and the final classification function $\mathrm{fr}=\beta 1 \mathrm{fl}+\beta \mathrm{rfr}$ is obtained.

- Test vector $\mathrm{x} \varepsilon \mathrm{Rq}$ is classified into the label maximizing the final function $\operatorname{Fr}(\mathrm{x}, \mathrm{k})$ with respect to $\mathrm{k} \varepsilon$ G.By applying IOOPL matching to each shadow, homogeneous sections that specify objects of the same category in different lighting conditions are obtained.

\section{2) Support Vector Machine}

Support vector machine have often been to provide better classification result the other widely used pattern recognition method such as like hood. The support vector machine are very attractive for the classification of remotely sensed data.

In gradients Training set $\mathrm{J}=\{\mathrm{xi}, \mathrm{yi}\} \mathrm{i}=\lambda \mathrm{i}$...m

$\mathrm{xi}$ is structured input

yi is structure output

$\lambda \mathrm{i}$ is a graph labelling

Future Function

$\phi(\mathrm{x}, \mathrm{y})$ : express relation between $\mathrm{x}$ and $\mathrm{y}$ has constant dimensionality. $\phi(x, y)$ may be a pair of image. Set of active/in active association $=m \varepsilon i=\wedge$ distantancei .yi.

This method has rectified the problem of not being able to obtain the inner and outer out lines of the minor.

\section{MERITS}

- Method can obtain a combatively complete shadow outline from a complex scene and derive the shadow-free image.

- To recover the shadow removal method based on IOOPL matching.

High performance and efficiency.

C. Result and Discussion

The experimental results revealed the following.

-The shadow detection method proposed in this paper can stably and accurately identify shadows.

-Threshold selection and false shadow removal can be conducted in simple but effective ways to ensure shadow detection accuracy.

-Compared with pixel-level detection, the object-oriented shadow detection method proposed in this paper can make full use of the spatial information of an image and can effectively rule out speckles and false shadows in the detection result. However, it is difficult to segment the small size shadows into an independent object, which will cause errors. 
-The shadow removal method based on IOOPL matching can effectively restore the information in a shadow area. The homogeneous sections obtained by IOOPL matching can show the radiation grey scale of the same object in a shadow area and a non-shadow area. The parameters calculated by using the radiation difference between inner and outer homogeneous sections can retrieve a shadow very effectively.

The two shadow removal strategies (AB and SVM) are both suitable for high-resolution urban remote sensing images. Moreover, there are advantages to each strategy:

AB can restore the texture details well while SVM has a more stable background radiance.

Further improvements are needed in the following ways. 1) Although image segmentation considering shadows can have better segmentation results, insufficient segmentation still exists. For example, a black car and its shadow cannot be separated. Also, parts of the shadow from low trees cannot be separated from the leaves.

2) Because of the filming environment or some other reasons, obvious colour cast can be seen in some parts of a shadow area. IOOPL matching could relieve this case to a certain extent but not completely resolve the problem.

\section{VI.CONCLUSION}

In order to get a shadow detection result, image segmentation considering shadows is applied first. Then, suspected shadows are selected through spectral features and spatial information of objects, and false shadows are ruled out. The subsequent shadow detection experiments compared traditional image segmentation and the segmentation considering shadows, as well as results from traditional pixel-level threshold detection and objectoriented detection. Meanwhile, they also show the effects of different steps with the proposed method.

For shadow removal, after the homogeneous sections have been obtained by IOOPL matching, we put forward two strategies: relative radiation correction for the objects one at a time, and removal of all shadows directly after PF is applied to all the Homogeneous sections and correction parameters are obtained.

\section{ACKNOWLEDGEMENT}

I would like to thank my guide Prof. K.Selvam Asst.Prof.Computer Science and Engineering Department, Parisutham Institute of Technology and Science, Thanjavur for his help and guidance to enable me to propose this system

\section{REFERENCES}

1] S. Ji and X. Yuan, "A method for shadow detection and change detection of man-made objects," Remote Sens., vol. 11, no. 3, pp. 323-329, 2007.

[2] G. Finlayson, S. Hordley, and M. Drew, "Removing shadows from images," in Proc. ECCV, May 28-31, 2002, pp. 823-836, Vision-Part IV.

[3] K.-L. Chung, Y.-R. Lin, and Y.-H. Huang, "Efficient shadow detection of colour aerial images based on successive thresholding scheme," IEEE Trans. Geosci. Remote Sens., vol. 47, no. 2, pp. 671-682, Feb. 2009.

4] H. Ma, Q. Qin, and X. Shen, "Shadow segmentation and compensation in high resolution satellite images," in Proc. IEEE IGARSS, Jul. 2008, vol. 2, pp. 1036-1039.

[5] K. Sun, D. Li, and H. Sui, "An object-oriented image smoothing algorithm based on the convexity model and multi-scale segmentation," Geomatics Inf. Sci. Wuhan Univ., vol. 34, no. 4, pp. 423-426, 2009

[6] Z. Zhu and C. E. Woodcock, "Object-based cloud and cloud shadow detection in Landsat imagery," Remote Sens. Environ., vol. 118, pp. 83-94, 2012.

[7] A. Makarau, R. Richter, R. Muller et al., "Adaptive shadow detection using a blackbody radiator model," IEEE Trans. Geosci. Remote Sens., vol. 49, no. 6, pp. 2049-2059, 2011.

[8] L. Lorenzi, F. Melgani, and G. Mercier, "A complete processing chain for shadow detection and reconstruction in VHR images," IEEE TransGeosci. Remote Sens., vol. 50, no. 9, pp. 3440-3452, 2012.

[9] P. Sarabandi, F. Yamazaki, M. Matsuoka et al., "Shadow detection and radiometric restoration in satellite high resolution images," in Proc. IEEE IGARSS, Sep. 2004, vol. 6, pp. 3744-3747.

[10] J. S.-P. Shu and H. Freeman, "Cloud shadow removal from aerial photographs," Pattern Recog, vol. 23, no. 6, pp. 647-656, 1990.

\section{BIOGRAPHY}

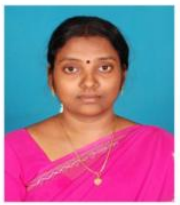

G.Gayathri received M.C.A from Ponnaiyah Ramajayam College in 2008. She is currently persuading M.E Computer Science in Parisutham Institute of Technology and Science 